NT@UW-02-023

\title{
The Quark-Mass Dependence of Two-Nucleon Systems
}

\author{
Silas R. Beane and Martin J. Savage \\ Department of Physics, University of Washington, \\ Seattle, WA 98195
}

\begin{abstract}
We explore the quark-mass dependence of two-nucleon systems. Allowed regions for the scattering lengths in the ${ }^{1} S_{0}$ and ${ }^{3} S_{1}$ channels as functions of the light-quark masses are determined from the current uncertainty in stronginteraction parameters that appear at next-to-leading order in the effective field theory. Where experimental constraints are absent, as is the case for the quark-mass dependent four-nucleon operators, we use naive dimensional analysis. We find it likely that there is no bound state in the ${ }^{1} S_{0}$ channel in the chiral limit. However, given the present uncertainties in strong-interaction parameters it is unclear whether the deuteron is bound or unbound in the chiral limit.
\end{abstract}




\section{INTRODUCTION}

An important aspect of strong-interaction physics that is only now being explored is the dependence of nuclei and nuclear reactions on the fundamental parameters of nature, such as $\Lambda_{\mathrm{QCD}}$ and the quark masses, $m_{q}$. With recent developments in the effective field theory (EFT) description of multi-nucleon systems fundamental questions about nuclear physics can be addressed. While the $m_{q}$-dependence of the nuclear force is unrelated to present day observables, it is a fundamental aspect of nuclear physics, and in some sense serves as a benchmark for the development of a perturbative theory of nuclear forces. Having this behavior under control will be essential to any bridge between lattice QCD simulations and nuclear physics in the near future. In work by Bedaque, van Kolck and the authors [1] (which we will refer to as BBSvK), a new power-counting scheme, BBSvK-counting, was introduced, and with a partial next-to-leading order (NLO) calculation in this new counting scheme, an attempt was made to determine the behavior of the two-nucleon force as the $m_{q}$ were increased or decreased from their physical values. Calculations of the $m_{q}$-dependence of scattering in the ${ }^{1} S_{0}$-channel and ${ }^{3} S_{1}-{ }^{3} D_{1}$ coupled-channels as well as a discussion of the deuteron binding energy were presented. In a subsequent paper [2] we performed the first complete NLO calculation in BBSvK-counting of the $m_{q}$-dependence of the two-nucleon systems. Previous estimates of such $m_{q}$-dependences $[3,4]$ that served as input to place bounds on physics beyond the standard model via the time-variation of fundamental parameters on cosmological time scales were found to be incomplete.

In this work, which should be considered a sequel to Ref. [2], we investigate the $m_{q^{-}}$ dependence of the two-nucleon systems, incorporating the present uncertainties in strong interaction parameters ${ }^{*}$. In some instances, parameters that play a central role in the $m_{q}$-dependence, such as the leading $m_{q}$-dependent four-nucleon interaction, are not constrained experimentally. In such cases, we use naive-dimensional-analysis (NDA) to provide a "reasonable range" for their values. Of particular interest to us is the behavior of the two-nucleon sector in the chiral limit. We find it likely that there is no di-nucleon bound state in the ${ }^{1} S_{0}$-channel; i.e. the scattering length remains negative. On the other hand, we cannot determine if there is a bound-state in the ${ }^{3} S_{1}-{ }^{3} D_{1}$ coupled channels in the chiral limit. The strong interaction parameters are sufficiently uncertain at present to preclude a definitive answer to this fundamental question.

\section{THE ${ }^{1} S_{0}$-CHANNEL AND THE DI-NUCLEON}

In the ${ }^{1} S_{0}$-channel, pions give a subleading contribution to the scattering amplitude and are included in perturbation theory. In this channel, BBSvK-counting is equivalent to Kaplan-Savage-Wise (KSW) power-counting [5]. Thus, analytic expressions for the scattering amplitude, and hence the scattering length, as a function of $m_{q}$ and momentum, $p$, are

straightforwardly found. From the NLO amplitude [5] it is easy to construct $p \cot \delta^{\left({ }^{1} S_{0}\right)}$,

*We are grateful to J. Bjorken who suggested this work to us during Marshall Baker Symposium held at the UW in June 2002. 
which has a well-behaved power-series expansion for $p<m_{\pi} / 2$, and thus a linear combination of $C_{0}$ (the coefficient of the $m_{q^{-}}$and $p$-independent four-nucleon operator) and $D_{2}$ (the coefficient of the $p$-independent and leading $m_{q}$-dependent four-nucleon operators) can be determined in terms of the scattering length $a^{\left({ }^{1} S_{0}\right)}=-23.714 \pm 0.013 \mathrm{fm}$ at the physical value of the pion mass. Furthermore, $C_{2}$ (the coefficient of the $m_{q}$-independent, leading $p^{2}$-dependent four-nucleon operator) can be determined in terms of the effective range, $r^{\left({ }^{1} S_{0}\right)}=2.73 \pm 0.03 \mathrm{fm}$. Once these parameters are fixed, the scattering length, effective range and phase-shift can be determined as a function of $m_{q}$ at NLO in the KSW expansion. The scattering length is, in the isospin limit,

$$
\frac{1}{a^{\left(1 S_{0}\right)}}=\gamma+\frac{g_{A}^{2} M_{N}}{8 \pi f_{\pi}^{2}}\left[m_{\pi}^{2} \log \left(\frac{\mu}{m_{\pi}}\right)+\left(\gamma-m_{\pi}\right)^{2}-(\gamma-\mu)^{2}\right]-\frac{M_{N} m_{\pi}^{2}}{4 \pi}(\gamma-\mu)^{2} D_{2}(\mu)
$$

where $\gamma=\mu+4 \pi / M_{N} C_{0}(\mu)$, and $\mu$ is the PDS renormalization scale [5]. We have used the leading order ( $\mathrm{LO})$ relation between the pion mass, $m_{\pi}$, and the light-quark masses, $m_{q}$. As pions are treated in perturbation theory, the $m_{q}$-dependence arising from the chiral expansions of $g_{A}, f_{\pi}$ and $M_{N}$ is formally higher order in KSW-counting and is neglected. The physical values of these parameters, $g_{A} \sim 1.25, f_{\pi} \sim 132 \mathrm{MeV}$ and $M_{N} \sim 939 \mathrm{MeV}$, are used for the analysis in this channel.

At present, the coefficient $D_{2}$ has not been experimentally separated from the coefficient $C_{0}$ in this channel, or in the ${ }^{3} S_{1}-{ }^{3} D_{1}$ coupled-channels. This is because both parameters are coefficients of momentum-independent operators with the only difference between the operators arising from their couplings to pions; the $C_{0}$ operator does not give rise to interactions between two-nucleons and multiple pions, while the $D_{2}$ operator does. In order to determine the range of values of $D_{2}$ consistent with NDA, we use the expression for the scattering length in eq. (1) at the physical value of the pion mass and find values of $C_{0}\left(m_{\pi}^{\mathrm{PHYS}}\right)$ and $D_{2}\left(m_{\pi}^{\text {PHYS }}\right)$ that satisfy the constraint

$$
\left|\left(m_{\pi}^{\text {PHYS }}\right)^{2} D_{2}\right|<\eta\left|C_{0}\right|
$$

where the constant $\eta$ is chosen to be $1 / 5$ and $1 / 15$ for demonstrative purposes. It is worthwhile discussing why these values are chosen. Very roughly, $\eta \sim\left(m_{\pi} / \Lambda\right)^{2}$ where $\Lambda$ represents the scale at which the EFT description breaks down. Naively, the KSW perturbative-pion expansion breaks down at a scale $\Lambda_{\mathrm{NN}}=8 \pi f_{\pi}^{2} / g_{A}^{2} M \sim 300 \mathrm{MeV}$. This would then give $\eta \sim 1 / 5$. On the other hand, one might expect that a better estimate of the cutoff scale is given by the mass of the fictitious scalar, $\sigma$, that provides intermediate-range attraction in many potential models. Taking $m_{\sigma} \sim 500-600 \mathrm{MeV}$ one then arrives at $\eta \sim 1 / 15$. Of course this last argument is somewhat specious; it is really at $m_{\sigma} / 2 \sim \Lambda_{\mathrm{NN}}$ that $\sigma$ should be treated as a dynamical object. This discussion underscores the difficulty in providing an a priori estimate of a parameter in an EFT. Hence, given the complete lack of experimental information on $D_{2}$, and the absence of any reliable method to estimate its value, we take $\eta$ to be $1 / 5$ and $1 / 15$ to provide an estimate of the uncertainty arising from $D_{2}$. We stress that even larger values of $\eta$, such as $1 / 3$, are not excluded experimentally. Of course larger values of $\eta$ lead to a smaller radius of convergence of the EFT. In Fig. 1 we show the scattering length in the ${ }^{1} S_{0}$-channel as a function of $m_{q}$, where we have randomly sampled values of 


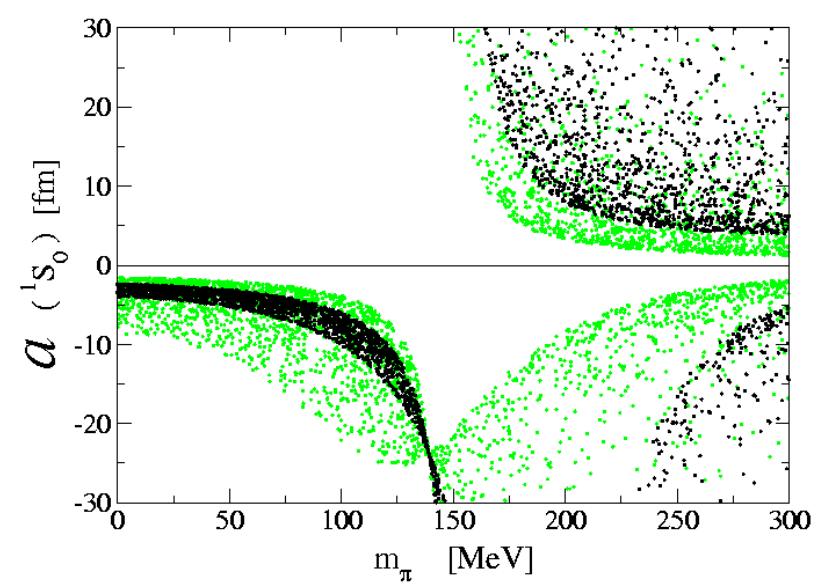

FIG. 1. The scattering length in the ${ }^{1} S_{0}$-channel as a function of the pion mass. The light gray region corresponds to $\eta=1 / 5$ and the black region corresponds to $\eta=1 / 15$. At the physical value of the pion mass the scattering length is $a^{\left({ }^{1} S_{0}\right)}=-23.714 \pm 0.013 \mathrm{fm}$.

$m_{\pi}$ and $\eta$ within the allowed ranges ${ }^{\dagger}$. From the standpoint of NDA, it is likely that the di-neutron is unbound in the chiral limit for any values of $m_{q}$ less than the physical values. Of course, the same will be true for the other di-nucleon states in the ${ }^{1} S_{0}$-channel by isospin symmetry. On the other hand, for $m_{q}$ larger than their physical values, we see that there are both bound and unbound di-neutrons for values of the strong-interaction parameters consistent with NDA. This observation reinforces our previous analysis [2].

For the strong-interaction parameter sets where the scattering length remains unnaturally large in the chiral limit, it is likely that higher-order contributions to the scattering amplitude will significantly disturb the system. Thus, for such situations, higher-order contributions may lead to a bound state where one does not exist at NLO.

\section{THE ${ }^{3} S_{1}-{ }^{3} D_{1}$ COUPLED-CHANNELS AND THE DEUTERON}

The deuteron resides in the ${ }^{3} S_{1}-{ }^{3} D_{1}$ coupled-channels when the $m_{q}$ assume their physical values. In our previous work $[1,2]$ we found that insufficient is presently known about the strong-interaction couplings in the two-nucleon sector to determine if the deuteron is bound or unbound for small values of $m_{q}$, including the chiral limit, or for $m_{q}$ larger than their physical values. In this section we make these findings more explicit and determine the regions allowed by data, and by NDA in the absence of data, for the ${ }^{3} S_{1}$ scattering length and deuteron binding energy, $B_{d}$, as a function of $m_{q}$, at NLO in BBSvK-counting.

The details of the calculation of scattering lengths, phase shifts and bound state energies in the ${ }^{3} S_{1}-{ }^{3} D_{1}$ coupled channels can be found in Refs. [1,2], and we do not repeat the details

\footnotetext{
${ }^{\dagger}$ We choose to use scatter plots as the point density reflects the probability associated with a particular set of low-energy constants.
} 
here. However, a brief description of the procedure, and of the sources of uncertainty is in order. In this channel, unlike the ${ }^{1} S_{0}$-channel, the chiral limit of one-pion-exchange (OPE) is included at LO in BBSvK-counting. This then allows for the re-summation of the large tensor-force that persists in the chiral limit, and is responsible for the non-convergence of KSW-counting in this channel [6]. Deviations from the chiral limit are included in perturbation theory, as are higher-dimension operators and multiple pion exchanges [1]. In the ${ }^{3} S_{1}-{ }^{3} D_{1}$ coupled-channels, OPE generates both central and tensor potentials,

$$
V_{C}^{(\pi)}\left(r ; m_{\pi}\right)=-\alpha_{\pi} m_{\pi}^{2} \frac{e^{-m_{\pi} r}}{r} \quad, \quad V_{T}^{(\pi)}\left(r ; m_{\pi}\right)=-\alpha_{\pi} \frac{e^{-m_{\pi} r}}{r}\left(\frac{3}{r^{2}}+\frac{3 m_{\pi}}{r}+m_{\pi}^{2}\right)
$$

where $\alpha_{\pi}=g_{A}^{2}\left(1-2 m_{\pi}^{2} \bar{d}_{18} / g_{A}\right)^{2} /\left(8 \pi f_{\pi}^{2}\right)$. The low-energy constant (LEC) $\bar{d}_{18}$ has been determined through various fitting procedures [7-10] to be $-0.78 \pm 0.27,-0.83 \pm 0.06$, $-1.30 \pm 0.24[8,9]$ and $-10.14 \pm 0.45 \mathrm{GeV}^{-2}[10]$ at third-order in the chiral expansion. While we are unable to establish a definition of the uncertainties associated with each of these determinations, we assume that they represent $1 \sigma$ errors arising from a parabolic error analysis of the MINUIT package, as partially described in Ref. [10]. Further, in the absence of an error correlation matrix, we will treat the uncertainties in the values of LECs as uncorrelated; this constitutes a weakness in our propagation of errors. Thus, we sample values of $\bar{d}_{18}$ over the range $-1.54 \mathrm{GeV}^{-2}<\bar{d}_{18}<-0.51 \mathrm{GeV}^{-2}$, corresponding to the $1 \sigma$ limits of the first three determinations above ${ }^{\ddagger}$, and as discussed in Ref. [9], this range is probably an underestimate of the true uncertainty in $\bar{d}_{18}$. A fourth-order calculation has been performed and extracted values of $\bar{d}_{18}$ that are consistent with the three values above [9].

As the chiral limit of the potentials in eq. (3) contribute at LO, the leading $m_{q^{-}}$ dependence of $g_{A}, f_{\pi}$ and $M_{N}$ are required at NLO, as discussed in BBSvK. Each of these observables has been studied extensively, the results of which can be found in Refs. [7,11-13], and up to next-to-next-to-leading order (NNLO) it is known that

$$
\begin{aligned}
f_{\pi} & =f_{\pi}^{(0)}\left[1-\frac{1}{4 \pi^{2}\left(f_{\pi}^{(0)}\right)^{2}} m_{\pi}^{2} \log \left(\frac{m_{\pi}}{m_{\pi}^{\mathrm{PHYS}}}\right)+\frac{m_{\pi}^{2}}{8 \pi^{2}\left(f_{\pi}^{(0)}\right)^{2}} \bar{l}_{4}\right] \\
M_{N} & =M_{N}^{(0)}-4 m_{\pi}^{2} c_{1} \\
g_{A} & =g_{A}^{(0)}\left[1-\frac{2\left(g_{A}^{(0)}\right)^{2}+1}{4 \pi^{2}\left(f_{\pi}^{(0)}\right)^{2}} m_{\pi}^{2} \log \left(\frac{m_{\pi}}{m_{\pi}^{\mathrm{PHYS}}}\right)-\frac{\left(g_{A}^{(0)}\right)^{2} m_{\pi}^{2}}{8 \pi^{2}\left(f_{\pi}^{(0)}\right)^{2}}+\frac{4 m_{\pi}^{2}}{g_{A}^{(0)}} \bar{d}_{16}\right],
\end{aligned}
$$

where $\bar{l}_{4}=4.4 \pm 0.2[11,12], c_{1} \sim-1 \mathrm{GeV}^{-1}$ [7] are $m_{q}$-independent constants (we have explicitly separated the logarithmic contribution from $\bar{l}_{4}$ ) and the superscript $(0)$ denotes the chiral limit value. We use $g_{A}=1.25, M_{N}=\left(M_{n}+M_{p}\right) / 2$ and $f_{\pi}=135 \mathrm{MeV}$. The parameter in eq. (4) that is most uncertain and gives rise to a substantial uncertainty in our calculations in the two-nucleon sector is $\bar{d}_{16}$. A complete analysis by Fettes [9] of the $\pi N$ sector provides three different determinations of $\bar{d}_{16}:-0.91 \pm 0.74,-1.01 \pm 0.72$ and $-1.76 \pm 0.85 \mathrm{GeV}^{-2}$. Thus we sample this parameter over the range $-2.61 \mathrm{GeV}^{-2}<$

$\ddagger$ The value, $\bar{d}_{18} \sim-10 \mathrm{GeV}^{-2}[10]$ is significantly larger than the other determinations and we discard it. 
$\bar{d}_{16}<-0.17$, again corresponding to the $1 \sigma$ limits of the three determinations. In our previous paper [2], we retained only the chiral logarithm contribution to $g_{A}$, and evaluated its argument at the scale $\lambda=500 \mathrm{MeV}$, which corresponds to choosing $\bar{d}_{16} \sim+1 \mathrm{GeV}^{-2}$. Note that while this value is outside the $1 \sigma$ range of $\bar{d}_{16}$, it is not excluded by data. As $\bar{l}_{4}$ makes only a small contribution to the OPE potential, its uncertainty has negligible impact and we hold $\bar{l}_{4}$ fixed to its central value in what follows.

At NLO in BBSvK-counting there is a contribution from the chiral limit of two-pion exchange (TPE) and from an insertion of the $C_{2}$ and $D_{2}$ operators. The TPE potential in coordinate space has been computed in Ref. [14-16], and in the chiral limit becomes

$$
V_{C}^{(\pi \pi)}(r ; 0)=\frac{3\left(22 g_{A}^{4}-10 g_{A}^{2}-1\right)}{64 \pi^{3} f_{\pi}^{4}} \frac{1}{r^{5}} \quad, \quad V_{T}^{(\pi \pi)}(r ; 0)=-\frac{15 g_{A}^{4}}{64 \pi^{3} f_{\pi}^{4}} \frac{1}{r^{5}} .
$$

In order to regulate the singular potentials that occur at LO and NLO we use a spatial square-well of radius $R[1,2,17]$, where the potential outside the square well is

$$
\mathcal{V}_{L}\left(r ; m_{\pi}\right)=\left(\begin{array}{cc}
-M_{N} V_{C}\left(r ; m_{\pi}\right) & -2 \sqrt{2} M_{N} V_{T}\left(r ; m_{\pi}\right) \\
-2 \sqrt{2} M_{N} V_{T}\left(r ; m_{\pi}\right) & -M_{N}\left(V_{C}\left(r ; m_{\pi}\right)-2 V_{T}\left(r ; m_{\pi}\right)\right)-6 / r^{2}
\end{array}\right),
$$

where

$$
V_{C}\left(r ; m_{\pi}\right)=V_{C}^{(\pi)}\left(r ; m_{\pi}\right)+V_{C}^{(\pi \pi)}(r ; 0) \quad, \quad V_{T}\left(r ; m_{\pi}\right)=V_{T}^{(\pi)}\left(r ; m_{\pi}\right)+V_{T}^{(\pi \pi)}(r ; 0) .
$$

The potential inside the square well is

$$
\mathcal{V}_{S}\left(r ; m_{\pi}, k^{2}\right)=\left(\begin{array}{cc}
-M_{N}\left(V_{C_{0}}+V_{D_{2}} m_{\pi}^{2}+k^{2} V_{C_{2}}\right) & 0 \\
0 & -M_{N}\left(V_{C_{0}}+V_{D_{2}} m_{\pi}^{2}+k^{2} V_{C_{2}}\right)-6 / r^{2}
\end{array}\right)
$$

where $V_{C_{0}}, V_{D_{2}}$ and $V_{C_{2}}$ are constant potentials corresponding to the renormalized local operators with coefficients $C_{0}, D_{2}$ and $C_{2}$ in the ${ }^{3} S_{1}-{ }^{3} D_{1}$ coupled-channels, respectively , and again we have used the LO relation between the pion mass and $m_{q}$. As discussed in BBSvK, we can make an identification between the coefficients of local operators and the constant potentials of the square-wells that enter into eq. (8), e.g.

$$
C_{i} \delta^{(3)}(r) \rightarrow \frac{3 C_{i} \theta(R-r)}{4 \pi R^{3}} \equiv V_{C_{i}} \theta(R-r)
$$

and similarly for $D_{i}$ and $V_{D_{i}}$. It is important to recall that there is implicit $m_{q}$-dependence in this potential due to $g_{A}, M_{N}$ and $f_{\pi}$, in addition to the explicit dependence from $D_{2} m_{\pi}^{2}$, and from OPE. Defining the wavefunction $\Psi$ to be

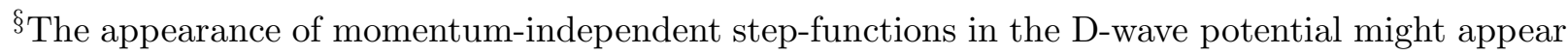
somewhat puzzling. However, this arises naturally when a spatial regulator is used to "smear out" the local short-distance part of the central potential in a manner that is consistent with rotational invariance. Contributions of this D-wave component to the scattering amplitude are suppressed by powers of the square-well radius, $R$, as $R \rightarrow 0$, as required in the renormalized theory.
} 


$$
\Psi(r)=\left(\begin{array}{c}
u(r) \\
w(r)
\end{array}\right)
$$

where $u(r)$ is the $\mathrm{S}$-wave wavefunction and $w(r)$ is the $\mathrm{D}$-wave wavefunction, the regulated Schrödinger equation is

$$
\Psi^{\prime \prime}(r)+\left[k^{2}+\mathcal{V}_{L}\left(r ; m_{\pi}\right) \theta(r-R)+\mathcal{V}_{S}\left(r ; m_{\pi}, k^{2}\right) \theta(R-r)\right] \Psi(r)=0
$$

It is this Schrödinger equation that we solve to generate the scattering length and location of the bound-state (if present) in this channel. A detailed comparison of phase shifts generated with this formulation, and the results of the Nijmegen partial wave analysis [18] can be found in Ref. [1,2].

A few words are in order concerning the power-counting. BBSvK-counting concerns itself with a consistent removal of cutoff effects, order-by-order in the EFT expansion [1]. The OPE potential is special in that only the chiral limit piece requires renormalization. This is because the $1 / r^{2}$ contribution in the pion mass expansion vanishes and all other terms in the expansion are regular at the origin. The chiral expansion of OPE has been shown to converge, albeit slowly, in Ref. [1]. We could, of course, arrange the power counting so as to keep as many orders in the OPE perturbative chiral expansion as necessary in order to match to the results shown in our paper, which treats the chiral expansion of OPE to all orders; however, this would be rather pointless, since treating the potential to all orders in the chiral expansion is perfectly innocuous from the perspective of renormalization, and in such cases it is perfectly legitimate to treat perturbative physics nonperturbatively. On the other hand, the TPE potential has a significantly more divergent chiral expansion as there are singular-attractive $1 / r^{5}$ and $1 / r^{3}$ contributions. It is likely that treating TPE to all orders while keeping only the counterterms at NLO in Weinberg counting does not remove all cutoff artifacts (scaling as logarithms or inverse powers of the coordinate-space cutoff). We have confirmed this strong cutoff dependence of the full TPE numerically; this is, in fact, strong motivation for using BBSvK counting.

Using the same definition of $\eta$ as in eq. (2), we generate values for $D_{2}$ and $C_{0}$ consistent with NDA, and solve the Schrödinger equation in eq. (11) for the scattering length in the ${ }^{3} S_{1}$ channel and the deuteron binding energy. We choose a cutoff of $R=0.45 \mathrm{fm}$. The cutoffindependence of our results is discussed in Refs. [1,2]. In Fig. 2, the scattering length in the ${ }^{3} S_{1}$ channel is shown as a function of the pion mass, where we have used the LO relation between $m_{q}$ and $m_{\pi}$. Again we have chosen $\eta$ to have the values $\eta=1 / 5$ and $1 / 15$ to illustrate the effect of $D_{2}$. We take $\bar{d}_{18}=-1.54 \mathrm{GeV}^{-2}$ and $-2.61 \mathrm{GeV}^{-2}<\bar{d}_{16}<-0.17 \mathrm{GeV}^{-2}[9]$. We again randomly and uniformly sample parameter space within the allowed range**. In Fig. 3 the scattering length in the ${ }^{3} S_{1}$ channel is shown as a function of the pion mass. The difference with Fig. 2 is that in Fig. 3 we take $\bar{d}_{18}=-0.51 \mathrm{GeV}^{-2} \dagger$. In Fig. 4 we show

\footnotetext{
**A more appropriate procedure might be to sample $\bar{d}_{16}$ from a Gaussian-weighted distribution.

${ }^{\dagger \dagger}$ While $\bar{d}_{18}$ does not contribute in the chiral limit, it does alter the strength of the nucleon-nucleon force at the physical value of the pion mass, where the local four-nucleon operators are fit to data. Data, of course, contains all orders in the chiral expansion.
} 


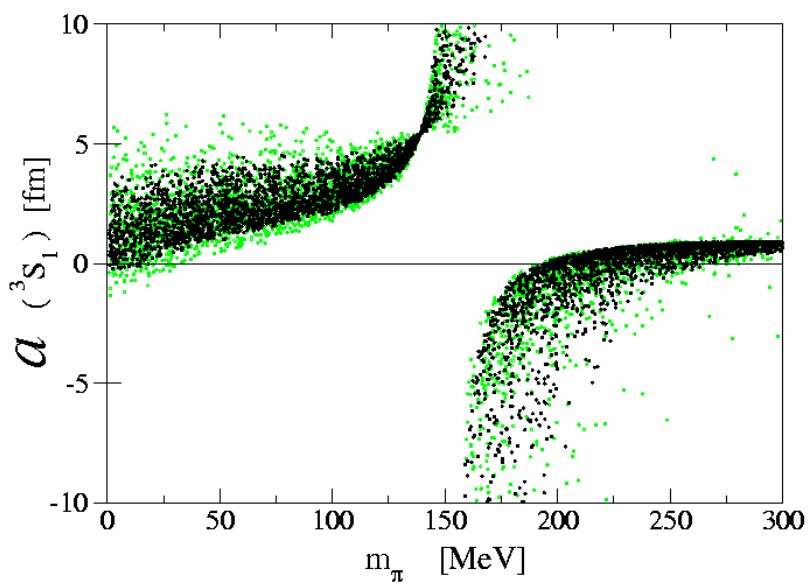

FIG. 2. The scattering length in the ${ }^{3} S_{1}$-channel as a function of the pion mass. The light shaded region corresponds to $\eta=1 / 5$ and the black region corresponds to $\eta=1 / 15$. The parameter $\bar{d}_{16}$, defined in eq. (4), is taken to be in the interval $-2.61 \mathrm{GeV}^{-2}<\bar{d}_{16}<-0.17 \mathrm{GeV}^{-2}[9]$, and $\bar{d}_{18}=-1.54 \mathrm{GeV}^{-2}$. At the physical value of the pion mass the scattering length is $a^{\left({ }^{3} S_{1}\right)} \sim+5.425 \mathrm{fm}$.

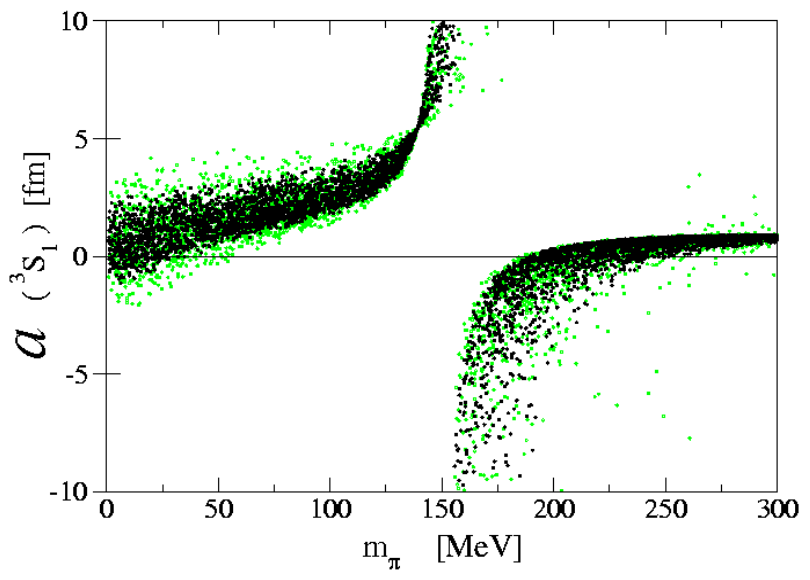

FIG. 3. The scattering length in the ${ }^{3} S_{1}$-channel as a function of the pion mass. Parameters and labels are the same as Fig. 2 except $\bar{d}_{18}=-0.51 \mathrm{GeV}^{-2}$. 


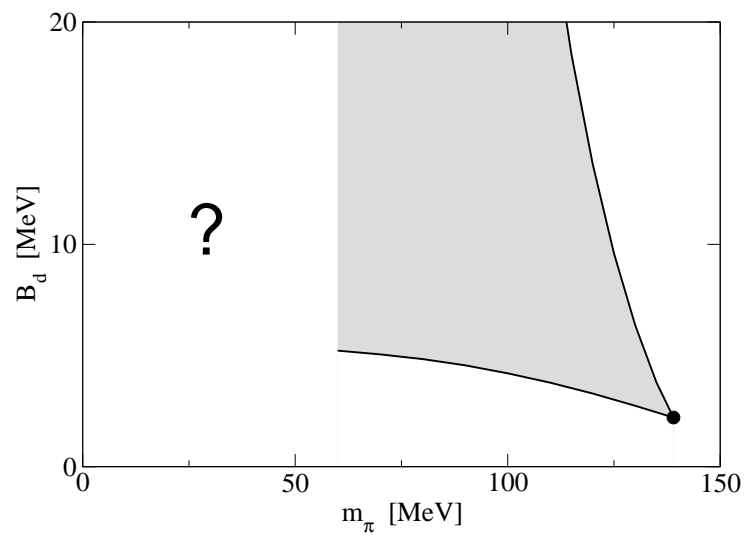

FIG. 4. The deuteron binding energy as a function of the pion mass (from the physical value to the chiral limit). We do not show values below $m_{\pi}=60 \mathrm{MeV}$ since the deuteron can be both bound and unbound. The shaded region corresponds to $\eta=1 / 5,-2.61 \mathrm{GeV}^{-2}<\bar{d}_{16}<-0.17 \mathrm{GeV}^{-2}$, and $-1.54 \mathrm{GeV}^{-2}<\bar{d}_{18}<-0.51 \mathrm{GeV}^{-2}$.

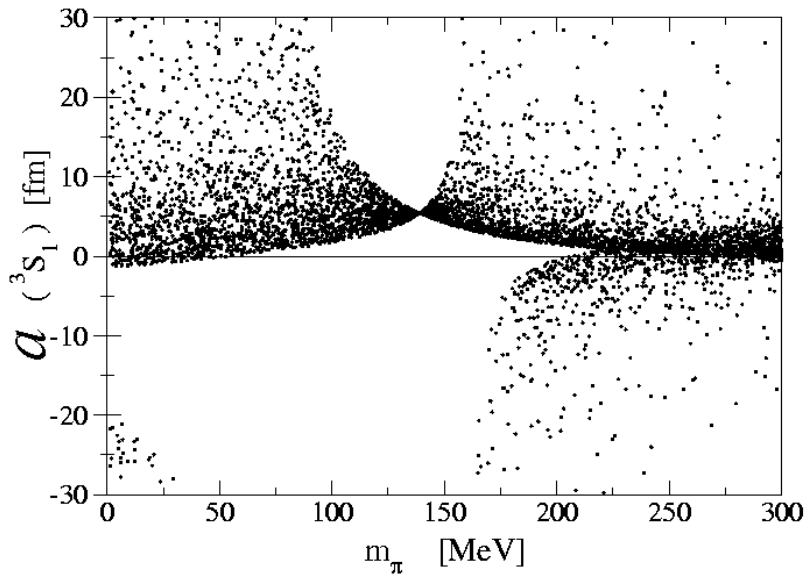

FIG. 5. The scattering length in the ${ }^{3} S_{1}$-channel as a function of the pion mass for $\bar{d}_{16}=+1 \mathrm{GeV}^{-2}, \eta=1 / 3$ and $\bar{d}_{18}=-0.51 \mathrm{GeV}^{-2}$. 
the deuteron binding energy as a function of the pion mass for the full range of $\bar{d}_{18}$ and $\bar{d}_{16}$ and taking $\eta=1 / 5$.

Perhaps it is worth interpreting the binding energy curves in Fig. 4 using the scattering length curves of Fig. 2 and Fig. 3. It is obvious from Fig. 2 and Fig. 3 that the scattering length can be negative for $m_{\pi} \lesssim 60 \mathrm{MeV}$, indicating an unbound deuteron. Naively one would expect the scattering length to go through infinity to unbind the deuteron; this would correspond to the deuteron binding energy going through zero. However, in this work we find that the deuteron becomes unbound in the approach to the chiral limit by becoming increasingly deeply bound and thus leaving the range of applicability of the EFT.

From these figures we conclude that the present uncertainty in strong-interaction parameters precludes a definitive statement being made concerning the binding or non-binding of the deuteron in the chiral limit. This is consistent with our previous studies $[1,2]$. It is a combination of uncertainties in the $\pi N$ sector and the lack of any information in the two-nucleon sector regarding $D_{2}$ that conspire to prevent a definitive conclusion. It is clear that progress must be made on both issues before the chiral limit of the ${ }^{3} S_{1}-{ }^{3} D_{1}$ coupled channels can be predicted. To demonstrate the impact of $\bar{d}_{16}$, in Fig. 5 we show the scattering length in the ${ }^{3} S_{1}$ channel for $\bar{d}_{16}=+1 \mathrm{GeV}^{-2}$, a value that is not excluded by data. Clearly the sensitivity of the two-nucleon system to this parameter is rather dramatic. For this parameter set we see the deuteron unbinding by the scattering length going through both infinity and zero.

Impressive progress has been made in extrapolating lattice observables in the singlenucleon and meson sectors from lattice quark masses to the physical quark masses. In addition to being important in its own right, this work is essential for interpretation of lattice QCD results in nuclei. Extrapolations of some quenched lattice QCD observables at large quark masses $\left(m_{\pi} \sim 500 \mathrm{MeV}\right)$ to physical values of the quark masses are seen to be in good agreement with experimental values [19]. Future partially-quenched or unquenched simulations are required at smaller quark masses in order to have confidence in first principles calculations. As we discussed in Ref. [1,2], a quenched lattice calculation of the scattering lengths in both the ${ }^{1} S_{0}$ and ${ }^{3} S_{1}$-channels exists [20], but for pion masses greater than $\sim 550 \mathrm{MeV}$. While it would be tempting to extrapolate our results to these pion masses it is likely that the EFT will have broken down at much smaller pion masses. Moreover, as pointed out in Ref. [21], the long-distance part of the two-nucleon force is modified in quenched and partially-quenched QCD; for instance, long-distance Yukawa behavior is modified to pure exponential fall-off. This poses yet another challenge to bridging the gap between nuclear physics and lattice QCD [21].

\section{COMMENTS ON RECENT WORK BY EPELBAUM, GLÖCKLE AND MEIßNER}

While this paper was in preparation, an analysis by Epelbaum, Glöckle and Meißner (EGM) has become available [22] which also extends earlier work on this subject $[1,2]$. EGM consider the $m_{q}$-dependence of nuclear forces using their formulation of Weinberg's power-counting [23]. In contrast with our coordinate-space derivation, this work involves a numerical solution to the Lippmann-Schwinger equation with a potential generated order-byorder in the chiral expansion with a power-counting derived for the meson and single nucleon 
sectors. Their work is in qualitative agreement with the results of our previous papers $[1,2]$ and with the results we have presented here. Despite the differences in regularization method and the subtly different power-counting schemes, it not surprising to see that the two EFTs yield results that appear to be perturbatively close. In the ${ }^{1} S_{0}$-channel, it is encouraging to see that the results of the numerical calculations of Ref. [22] agree with our analytic results obtained from the perturbative-pion expansion. However, there are quite significant differences in the uncertainties associated with the extrapolation of the observables away from the physical value of the pion mass, particularly in the ${ }^{3} S_{1}-{ }^{3} D_{1}$ coupled channels. These discrepancies arise from a different treatment of uncertainties associated with the strong-interaction parameters $\bar{d}_{16}, \bar{d}_{18}$ and $D_{2}$. It appears that EGM [22] have chosen a range of values of $D_{2}$ consistent with $\eta \lesssim 1 / 19$, which we consider to be overly optimistic ${ }^{\ddagger \ddagger}$. EGM argue that, for the range of cutoffs they use to define their EFT, all experimentally determined low-energy constants in the two-nucleon sector fall within the "NDA" estimates that they have created. Unfortunately, at present there is no way to test the reliability of such estimates for $m_{q}$-dependent operators. Moreover, EGM have chosen not to propagate the known and relatively large errors associated with the extraction of $\bar{d}_{16}[9]$ from the single nucleon sector. In Fig. 6 we show the ${ }^{3} S_{1}$ scattering length as a function of the pion mass for the parameter ranges used by EGM. With their parameters we reproduce their results, up to terms higher order in both EFT descriptions. For instance, we recover their result of $1.25 \mathrm{fm} \lesssim a^{\left({ }^{3} S_{1}\right)} \lesssim 1.85 \mathrm{fm}$ in the chiral limit. Therefore, while we find the claims of Ref. [22] to be quite spectacular, e.g. a deuteron binding energy of $B_{d}^{(0)}=9.6 \pm 1.9_{-1.0}^{+1.8} \mathrm{MeV}$ in the chiral limit, it would appear that their error analysis is incomplete, and when a complete error analysis is performed, their claims are likely to be greatly diminished ${ }^{\S}$.

\section{CONCLUSIONS}

Understanding how nuclei and nuclear interactions depend upon the light-quark masses is a fundamental aspect of strong-interaction physics. We have been able to explore the $m_{q}$-dependence of two-nucleon systems using a recently-developed effective field theory and naive dimensional analysis. In the ${ }^{1} S_{0}$-channel we expect that di-nucleon systems, such as the di-neutron, are unbound for all values of $m_{q}$ less than their physical values. However, for $m_{q}$ larger than their physical values both bound and unbound systems are presently consistent

\footnotetext{
${ }^{\ddagger}$ We should note that EGM apply their NDA argument to the two operators $D_{S}$ and $D_{T}$. However, there are four operators, $D_{S 1, S 2}$ and $D_{T 1, T 2}$, that can be constructed with one insertion of $m_{q}$ [2]. With four operators present, we find that their method saturates the range over which NLO is a subleading correction, and thus reproduces the NDA estimates that we have used.

${ }^{\S}$ An addendum to Ref. [22] has recently been archived by EGM [24] which increases the error in the chiral limit of the deuteron binding energy by a factor $\sim 3: B_{d}^{(0)}=9.6_{-3.2-2.4-0.4}^{+4.4+5.7+0.6} \mathrm{MeV}$. While their results are becoming more consistent with our analysis, we believe that EGM continue to underestimate the errors in the strong interaction parameters. For instance, EGM have not implemented the error correlation matrix for parameters in the single-nucleon sector.
} 


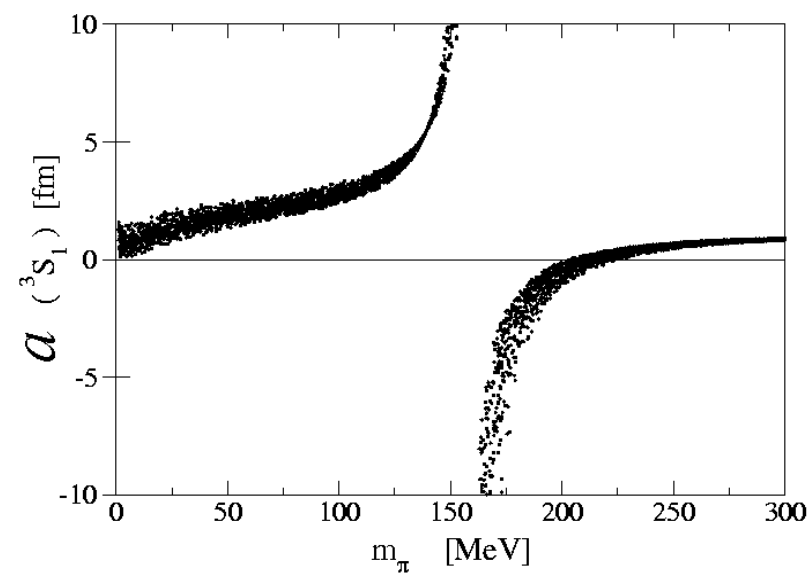

FIG. 6. The scattering length in the ${ }^{3} S_{1}$-channel as a function of the pion mass for the parameter set $-1.76 \mathrm{GeV}^{-2}<\bar{d}_{16}<-0.91 \mathrm{GeV}^{-2}, \bar{d}_{18}=-0.97 \mathrm{GeV}^{-2}$ and $\eta=1 / 20$ used in Ref. [22].

with data and NDA. In the ${ }^{3} S_{1}-{ }^{3} D_{1}$ coupled-channels, where the deuteron resides for the physical values of the quark masses, the deuteron may or may not be bound in the chiral limit. A more definitive statement can only be made with a more precise determination of the $\pi N$ coupling $\bar{d}_{16}$ and a determination of the coefficients of the leading $m_{q}$-dependent four-nucleon operators, $D_{2}$. As discussed in Ref. [2], it is likely that a determination of $D_{2}$ will require a future lattice QCD calculation. While we have not investigated higher partial waves, given that in this framework they are well described by perturbative pion exchange, we do not expect qualitative changes to their behavior away from the physical value of the pion mass. It is very exciting indeed to be so close to making fundamental statements about nuclear physics.

\section{Acknowledgments}

We thank the Benasque Center for Science where some of this work was performed. We thank J. Bjorken and U. van Kolck for stimulating discussions and we are grateful for useful conversations with Evgeni Epelbaum and U.-G. Meißner regarding the low-energy constants in the single-nucleon sector. This research was supported in part by the DOE grant DEFG03-97ER41014. 


\section{REFERENCES}

[1] S.R. Beane, P.F. Bedaque, M.J. Savage and U. van Kolck, Nucl. Phys. A700, 377 (2002).

[2] S.R. Beane and M.J. Savage, hep-ph/0206113.

[3] A. Bulgac, G.A. Miller and M. Strikman, Phys. Rev. C56, 3307 (1997).

[4] E.W. Kolb, M.J. Perry, T.P. Walker, Phys. Rev. D33, 869 (1986); J.D. Barrow, Phys. Rev. D35, 1805 (1987); V.V. Dixit and M. Sher, Phys. Rev. D37, 1097 (1988); B.A. Campbell and K.A. Olive, Phys. Lett. B345, 429 (1995); V. Agrawal, S.M. Barr, J.F. Donoghue, D. Seckel, Phys.Rev. D57, 5480 (1998); H. Oberhummer, R. Pichler and A. Csoto, nucl-th/9810057; Y. Fujii et al, hep-ph/9809549; T.E. Jeltema and M. Sher, Phys. Rev. D61, 017301 (1999); L. Bergstrom, S. Iguri and H. Rubinstein, Phys. Rev. D60, 045005 (1999); X. Calmet and H. Fritzsh, hep-ph/0112110; A. Csoto, H. Oberhummer and H. Schlattl, Nucl. Phys. A688. 560 (2001); Nucl. Phys. A689, 269 (2001); T. Chiba, gr-qc/0110118; P. Langacker, G. Segre, M.J. Strassler, Phys. Lett. B528, 121 (2002); T. Dent and M. Fairbairn, hep-ph/0112279; A.D. Dolgov, hep-ph/0201107; K. Ichikawa and M. Kawasaki, hep-ph/0203006; V.V. Flambaum and E.V. Shuryak, Phys. Rev. D65, 0103503 (2002); K.A. Olive, M. Pospelov, Y.-Z. Qian, A. Coc, M. Casse, and E. Vangioni-Flam, hep-ph/0205269.

[5] D.B. Kaplan, M.J. Savage, and M.B. Wise, Phys. Lett. B424, 390 (1998); Nucl. Phys. B534, 329 (1998).

[6] S. Fleming T. Mehen and I.W. Stewart, Nucl. Phys. A677, 313 (2000).

[7] U.-G. Meißner, Essay for the Festschrift in honor of Boris Ioffe, in the Encyclopedia of Analytic QCD, At the Frontier of Particle Physics, vol. 1, 417-505, edited by M. Shifman (World Scientific); hep-ph/0007092.

[8] N. Fettes, V. Bernard and U.-G. Meißner, Nucl. Phys. A669, 269 (2000).

[9] N. Fettes, JUL-3814 (PhD Thesis).

[10] N. Fettes and U.-G. Meißner, Nucl. Phys. A693, 693 (2001).

[11] J. Gasser and H. Leutwyler, Annals Phys. 158, 142 (1984).

[12] G. Colangelo, J. Gasser and H. Leutwyler, Nucl. Phys. B603, 125 (2001)

[13] N. Fettes and U.-G. Meißner, Nucl. Phys. A676, 311 (2000).

[14] C. Ordoñez, L. Ray and U. van Kolck, Phys. Rev. C53, 2086 (1996); Phys. Rev. Lett. 72, 1982 (1994); Phys. Lett. B291, 459 (1992).

[15] J.L. Friar, Mod. Phys. Lett. A11, 3043 (1996).

[16] N. Kaiser, R. Brockmann and W. Weise, Nucl. Phys. A625, 758 (1997).

[17] D.W.L. Sprung, W. van Dijk, E. Wang, D.C. Zheng, P. Sarriguren, and J. Martorell, Phys. Rev. C49, 2942 (1994).

[18] V.G.J. Stoks, R.A.M. Klomp, M.C.M. Rentmeester, and J.J. de Swart, Phys. Rev. C48, 792 (1993).

[19] See, for example, K. Orginos [RBC Collaboration], hep-lat/0209137; W. Detmold, W. Melnitchouk and A.W. Thomas, Phys. Rev. D66, 054501 (2002).

[20] M. Fukugita, Y. Kuramashi, M. Okawa, H. Mino and A. Ukawa, Phys. Rev. D52, 3003 (1995).

[21] S.R. Beane and M.J. Savage, Phys. Lett. B535, 177 (2002).

[22] E. Epelbaum, U. G. Meißner and W. Glöckle, nucl-th/0207089. 
[23] S. Weinberg, Phys. Lett. B251, 288 (1990); Nucl. Phys. B363, 3 (1991); Phys. Lett. B295, 114 (1992).

[24] E. Epelbaum, U. G. Meißner and W. Glöckle, nucl-th/0208040. 